\title{
Physics potential of Hyper-Kamiokande for neutrino oscillation measurements
}

John Walker*广

University of Winnipeg/TRIUMF

E-mail: jmgwalker@triumf.ca

Hyper-Kamiokande (Hyper-K) is a next generation underground water Cherenkov neutrino detector. It will serve as a far detector for a long baseline neutrino oscillation experiment using the J-PARC neutrino beam, with sensitivity to exclude $\mathrm{CP}$ conservation over a range of parameter space. Hyper-K has a broad physics program, with further sensitivity to proton decay, atmospheric neutrinos, and neutrinos from astronomical sources. The experimental set-up will be described as well as the physics potential, with particular reference to the sensitivity for CP violation.

The 21st international workshop on neutrinos from accelerators (NuFact2019)

August 26 - August 31, 2019

Daegu, Korea

\footnotetext{
*Speaker.

${ }^{\dagger}$ On behalf of the Hyper-Kamiokande collaboration.
} 


\section{Introduction}

Hyper-Kamiokande (Hyper-K) is a next generation underground water Cherenkov neutrino experiment [1] for the study of neutrino oscillations, astrophysical neutrinos, and nucleon decay. The J-PARC proton accelerator will fire a $30 \mathrm{GeV}$ proton beam at a carbon target creating a beam of neutrinos. The far detector for Hyper-K will have a $260 \mathrm{kton}$ volume observed by $\sim 40,000$ $50 \mathrm{~cm}$ high quantum efficiency photomultiplier tubes (PMTs), and be located at a baseline of $295 \mathrm{~km}$ and 2.5 degrees off-axis so that the beam is centred on the first oscillation maximum. This represents an increase in fiducial volume of $\sim 8$ times that of Super-Kamiokande (Super$\mathrm{K})$ [2] and, in combination with the upgraded beam power from $500 \mathrm{~kW}$ to $1.4 \mathrm{MW}$, Hyper-K will accumulate statistics $\sim 20$ times faster.

A suite of near detectors are proposed in order to constrain the systematic uncertainty enough to take advantage of the statistical improvements. Hyper-K will require an on-axis detector to monitor the beam direction and event rate, for which the T2K INGRID detector [3] can be repurposed. An upgraded version of the T2K ND280 near detector, an off-axis magnetized tracker, is also required to achieve charge separation to measure the wrong sign background, as well as provide a flux constraint, and to study the recoil system.

It is also proposed to build an off-axis angle spanning intermediate water Cherenkov detector (IWCD) [4], which provides a number of benefits. IWCD can measure the beam $v_{e}\left(\bar{v}_{e}\right)$ intrinsic backgrounds, as well as make a measurement of the $v_{e}\left(\bar{v}_{e}\right)$ cross sections on water. For a chargeparity (CP) violation measurement the $v_{e}$ appearance is compared with the $\bar{v}_{e}$ appearance, so this is an important systematic control. A further benefit to IWCD is that it has the same water target as the far detector, which reduces the extrapolation systematic. The water can be doped with gadolinium to make a neutron multiplicity measurement, and can also measure the neutrino energy versus different observables to study nuclear interactions.

The instrumented portion of IWCD will be movable through a deep chamber in order to span a range of angles relative to the neutrino direction. The inner detector will be $8 \mathrm{~m}$ diameter and $6 \mathrm{~m}$ tall to contain up to $1 \mathrm{GeV}$ muons. A $1 \mathrm{~m}$ radius optically separated outer detector will also be instrumented to provide active shielding. The IWCD inner detector will be populated with multiPMT (mPMT) modules to provide photocoverage. The mPMTs contain an array of 19 3" PMTs, which improve the granularity and timing response over larger photosensors. One of the benefits of the mPMT modules is that each PMT images a different part of the tank, providing additional directional information. There are also plans to install 5000 mPMTs in Hyper-K.

\section{Hyper-K Oscillation Analysis}

Hyper-K is sensitive to neutrino oscillation parameters through a number of different measurements. Measurements of the $v_{\mu}\left(\bar{v}_{\mu}\right)$ survival probabilities are sensitive to $\sin ^{2} 2 \theta_{23}$ and $\Delta m_{32}^{2}$. Measurements of the $v_{e}\left(\bar{v}_{e}\right)$ appearance probabilities are sensitive to $\sin ^{2} \theta_{23}, \sin ^{2} 2 \theta_{13}$, and $\Delta m_{32}^{2}$ in the leading term, and sensitive to $\delta_{C P}$ in the sub-leading terms. Hyper-K is also sensitive to the mass ordering through the matter effect.

The $v_{e}\left(\bar{v}_{e}\right)$ appearance probabilities change as a function of neutrino energy depending on the value of $\delta_{C P}$, in particular the sub-leading $\delta_{C P}$ term has a different sign. Hyper-K is therefore sen- 
sitive to the CP-violating phase by observing the difference in the $v_{e}\left(\bar{v}_{e}\right)$ appearance probabilities. The maximum difference in appearance probability is $\pm 27 \%$ when $\delta_{C P}=-90$. For Hyper-K's baseline of $295 \mathrm{~km}$ the matter effect is relatively small compared to the CP effect.

Fig. 1 shows the sensitivity that Hyper-K will have to different values of $\delta_{C P}$. After 10 years of operation Hyper-K will have 76(57)\% coverage at the 3(5) $\sigma$ level. The precision with which $\delta_{C P}$ can be measured is $22^{\circ}$ for $\delta_{C P}= \pm 90^{\circ}$ and $7^{\circ}$ for $\delta_{C P}=0^{\circ}$ or $180^{\circ}$. This analysis considers only what can be constrained with the beam, but the sensitivity could be enhanced by combining with atmospheric data.
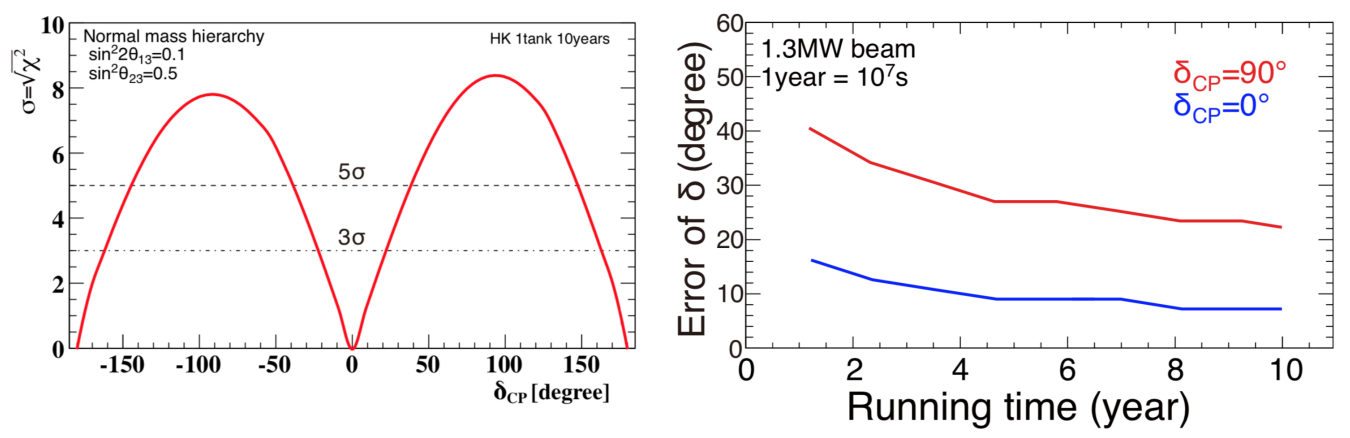

Figure 1: The sensitivity to exclude CP conservation over a range of values of $\delta_{C P}$ (left), and the precision with which $\delta_{C P}$ can be measured (right).

Primary cosmic ray interactions in the atmosphere produce a flux of atmospheric muon and electron neutrinos and their antineutrinos. These neutrinos have a wide energy range and a range of different flight lengths. The earth matter effect modifies the energy spectrum of oscillations as they pass through the Earth's core. The value of $\delta_{C P}$ affects the scale and direction of the atmospheric $v_{e}\left(\bar{v}_{e}\right)$ flux interference term at the order of $1 \mathrm{GeV}$. The octant of $\theta_{23}$ affects the magnitude of the multi-GeV resonance term. This resonance can be seen for $v_{e}$ appearance for the normal hierarchy case, but would be seen for $\bar{v}_{e}$ appearance for the inverted hierarchy.

Fig. 2 shows the sensitivity for rejecting the wrong hierarchy at $3 \sigma$ for all possible values of $\theta_{23}$ when combining the atmospheric and beam neutrinos in a joint fit analysis. For wrong octant rejection, Hyper-K has a greater than $3 \sigma$ sensitivity provided $\left|\theta_{23}-45^{\circ}\right| \geq 2.3^{\circ}$.

\subsection{Korean Detector Option}

A second tank in Korea [5] is being considered, which would have a number of benefits over a second tank at the same location as the far detector in Japan. There are a number of different candidate sites but the favoured one is underneath Mt. Bisul, which has a baseline of 1,088 km and located at an off-axis angle of $1.3^{\circ}$. The detector would therefore be located at the second oscillation maximum, which has an enhanced difference between $v_{e}$ and $\bar{v}_{e}$ appearance. Consequently the Korean detector would have a similar sensitivity to CP violation compared to the Japanese far detector even though the statistics are lower. Other benefits to a Korean detector would be increased sensitivity to the mass hierarchy because of the increased matter effect from the longer baseline and the higher neutrino energies. The Korean detector would also have more overburden than the Japanese detector so would have lower backgrounds for solar physics. 

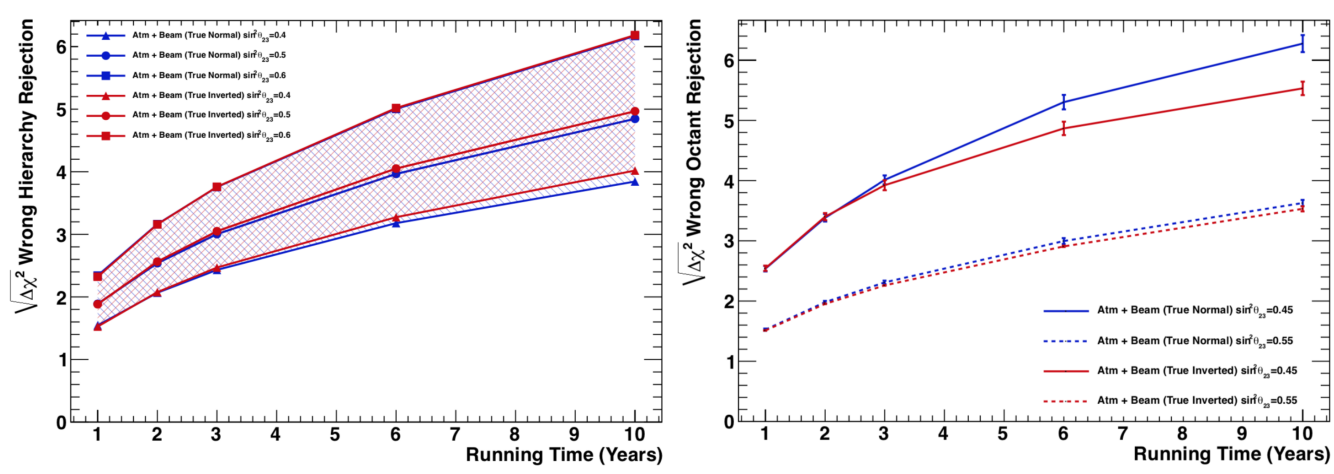

Figure 2: The sensitivity for wrong mass hierarchy rejection for all possible values of $\theta_{23}$ (left), and for wrong octant rejection for $\left|\theta_{23}-45^{\circ}\right| \geq 2.3^{\circ}$ (right).

Studies have shown that for a $\delta_{C P}$ measurement a second tank in Korea would have benefits not only over a single tank in Japan, but also over a second tank in Japan. The $\delta_{C P}$ measurement precision goes from $22^{\circ}$ for a single tank to $14^{\circ}$ when $\delta_{C P}=-90^{\circ}$, which is $3^{\circ}$ better than a second Japanese tank. The sensitivity for wrong mass hierarchy rejection goes from $4.5 \sigma$ to $9 \sigma$ when $\sin ^{2} \theta_{23}=0.5$.

\section{Controlling Systematic Errors}

For the $v_{e}\left(\bar{v}_{e}\right)$ appearance modes in the above studies, total systematic errors of 3.2\%(3.9\%) have been assumed. The current systematic assumed by T2K for the relative error on the forward and reverse horn current electron neutrino appearance is $\sim 6 \%$. Improvements to the experimental set-up are therefore necessary to reduce the systematic error to take advantage of the expected $3.2 \%$ statistical error. Furthermore, the error on the $v_{e} / \bar{v}_{e}$ cross-section ratio of $3 \%$ assumed by T2K is theoretically motivated and may be an underestimate, so a measurement should be made.

IWCD is well-suited to measure the $v_{e} / \bar{v}_{e}$ cross-section ratio because the relative $v_{e}\left(\bar{v}_{e}\right)$ flux increases as the detector goes further off-axis and so can make a direct measurement. A further benefit to IWCD is that the active shielding significantly reduces the $\gamma$ background. Studies have shown that, in the momenta of interest for Hyper-K, IWCD can achieve a $2 \%$ statistical uncertainty and a total uncertainty of $<4 \%$. The systematic error is dominated by the flux and cross-section modelling, however, so this can be improved through external flux measurements (such as by the EMPHATIC experiment) as well as improved cross-section modelling.

A potential source of asymmetry between $v_{e}$ and $\bar{v}_{e}$ appearance is the wrong-sign and intrinsic background. To achieve the target $1 \%$ systematic error contribution, the wrong-sign background must be measured to a $9 \%$ accuracy, and the intrinsic $v_{e}\left(\bar{v}_{e}\right)$ and neutral current background must be measured to a $3 \%$ accuracy. The wrong-sign background measurement can be achieved with the magnetized tracking detector, while the intrinsic background measurement can be made by IWCD. The spectra for intrinsic backgrounds at IWCD and Hyper-K are very similar, so only a small model dependent correction needs to be made. 
Another source of uncertainty is the neutrino interaction model. The reconstructed neutrino energy is calculated using an interaction model based on the final state lepton kinematics. However, non-CCQE processes tend to feed down to lower energies, an example being $2 \mathrm{p} 2 \mathrm{~h}$ interactions where the additional proton carries away some missing energy. At the oscillation maximum of $600 \mathrm{MeV}, \sim 60 \%$ of the events are from neutrinos with a true energy $>700 \mathrm{MeV}$. This feed-down effect can bias oscillation measurements if it is not properly modelled. By making measurements at a range of off-axis angles IWCD is able to make a unique constraint on this feed-down effect.

As IWCD observes the neutrino beam at different angles it accesses different energy spectra. Because neutrinos are produced from pion decays into muons and neutrinos, the more forwardgoing neutrinos have higher energies. Further off-axis angles therefore have lower energies and narrower distributions. This enables energy dependent measurements to be made. One such analysis technique is to take linear combinations of different flux slices to create a desired synthetic flux distribution. For instance a Gaussian flux could be made with a narrower distribution than that which is possible from a single off-axis flux slice. The reconstructed neutrino energy distributions for these pseudo-monochromatic beams then reveal the separation of the CCQE and non-CCQE scatters, whereas this effect is normally concealed by the width of the off-axis flux. A direct prediction of the effect of non-CCQE scatters on oscillation measurements can therefore be made, and thus IWCD provides a unique constraint on nuclear models.

Linear combinations can also be made to create other flux distributions, such as producing the oscillated flux at Hyper-K. Different oscillation hypotheses can therefore be tested by comparing the IWCD prediction to what is observed at Hyper-K. Because IWCD and Hyper-K have the same interaction material, and therefore the same cross section, the dependence on the neutrino interaction model is reduced.

\section{Summary}

Hyper-K is a next generation water Cherenkov detector which will study neutrino oscillations. It is capable of excluding CP conservation at the $5 \sigma$ level over a range of values of $\delta_{C P}$, as well as having sensitivity to the mass hierarchy and $\theta_{23}$ octant. There are plans to build a second tank in South Korea, which would improve the measurement sensitivities. Future long baseline oscillation experiments, such as Hyper-K, will be dominated by systematic rather than statistical uncertainty. IWCD is a novel near detector which will control many of the systematic errors.

\section{References}

[1] K. Abe et al., Hyper-Kamiokande Design Report, 1805.04163.

[2] Y. Fukuda et al., Evidence for Oscillation of Atmospheric Neutrinos, Phys. Rev. Lett. 81 (1998) 1562.

[3] K. Abe et al., The T2K experiment, Nuclear Instruments and Methods in Physics Research Section A: Accelerators, Spectrometers, Detectors and Associated Equipment 659 (2011) 106.

[4] S. Bhadra et al., Letter of Intent to Construct a nuPRISM Detector in the J-PARC Neutrino Beamline, 1412.3086.

[5] K. Abe et al., Physics potentials with the second Hyper-Kamiokande detector in Korea, Progress of Theoretical and Experimental Physics 2018 (2018) . 